\title{
Generic uniqueness of least area planes in hyperbolic space
}

\author{
BARIS COSKUNUZER
}

\begin{abstract}
We study the number of solutions of the asymptotic Plateau problem in $\mathbb{W}^{3}$. By using the analytical results in our previous paper, and some topological arguments, we show that there exists an open dense subset of $C^{3}$ Jordan curves in $S_{\infty}^{2}\left(\mathbb{H}^{3}\right)$ such that any curve in this set bounds a unique least area plane in $\mathbb{H}^{3}$.
\end{abstract}

53A10; 58B 15

\section{Introduction}

In this paper, we are interested in the number of solutions to the asymptotic Plateau problem. The asymptotic Plateau problem was solved by Michael Anderson in his seminal paper [1]. Furthermore in [2] he used geometric measure theory methods to prove that for any Jordan curve in $S_{\infty}^{2}\left(\mathbb{M}^{3}\right)$, there exists an embedded least area plane in $\mathbb{H}^{3}$ spanning that curve. Then, Hardt and Lin studied the asymptotic regularity of the least area surfaces in hyperbolic space in [6;14]. Also, Gabai [5] solved the asymptotic Plateau problem by using topological arguments developed by Hass and Scott [7]. Lang [9] generalized Anderson's methods to solve the problem in Hadamard Gromov hyperbolic spaces. In [3], the author solved the problem for Gromov hyperbolic spaces with cocompact metric by generalizing Gabai's techniques.

On the number of the least area planes spanning a given asymptotic curve, there are a few results so far. Anderson [1] showed that if the curve in $S_{\infty}^{2}\left(\mathbb{H}^{3}\right)$ bounds a convex domain in $S_{\infty}^{2}\left(\mathbb{M}^{3}\right)$, then there exists a unique least area plane in $\mathbb{M}^{3}$ spanning that curve. Then Lin [14] generalized this result to the curves bounding a star shaped domain in $S_{\infty}^{2}\left(\mathbb{M}^{3}\right)$. Recently, in [4], the author showed that for a generic $C^{3, \mu}$ Jordan curve in $S_{\infty}^{2}\left(\mathbb{M}^{3}\right)$, there exist finitely many least area planes in $\mathbb{H}^{3}$ asymptotic to that curve. In this paper, we improve the generic finiteness result of the previous paper [4] to a generic uniqueness result for a more general class of curves.

Here is an outline of the paper. In [4], we showed that the boundary restriction map $\pi$ from the space of minimal maps from $D^{2}$ to $\mathbb{H}^{3}$ with $C^{3}$ asymptotic data to the space of the $C^{3}$ immersions of $S^{1}$ into $S_{\infty}^{2}\left(\mathbb{H}^{3}\right)$ is Fredholm of index 0 . We consider the 
boundary restriction map $\pi$ from minimal maps to their boundary parametrizations. Since it is Fredholm of index 0, its derivative is an isomorphism for generic curves.

Fix a generic curve $\Gamma$ in $S_{\infty}^{2}\left(\mathbb{M}^{3}\right)$. By using the inverse function theorem, we find a neighborhood $U_{\Sigma}$ of a least area plane $\Sigma$ in $\pi^{-1}(\Gamma)$, mapping homeomorphically into a neighborhood $V_{\Gamma}$ of $\Gamma$. By taking a path $\alpha$ in $V_{\Gamma}$, and by considering the corresponding path $\pi^{-1}(\alpha)$ in $U_{\Sigma}$, we get a continuous family of minimal planes with disjoint asymptotic boundaries around $\Sigma$. Then, we show that this continuous family of minimal planes is indeed a foliation by least area planes of a neighborhood of $\Sigma$. This implies the uniqueness of the least area plane in $\mathbb{T}^{3}$ spanning $\Gamma$. Then we show the same result for any curve in a neighborhood of a generic curve, and we get an open dense subset of the $C^{3}$ Jordan curves in $S_{\infty}^{2}\left(\mathbb{M}^{3}\right)$ with the uniqueness result.

Main theorem Let $A$ be the space of $C^{3}$ simple closed curves in $S_{\infty}^{2}\left(\mathbb{M}^{3}\right)$. Then there exists an open dense subset $A^{\prime}$ in $A$ such that for any $\Gamma \in A^{\prime}$, there exists a unique least area plane $\Sigma$ with $\partial_{\infty} \Sigma=\Gamma$.

The organization of the paper is as follows. In the next section, we will give some preliminary results which will be used in the following sections. In Section 3, we will construct a foliated neighborhood of a least area plane spanning a generic curve. By using this, we will show that there is a unique least area plane spanning that curve. In Section 4, we will show that the curves with this property is indeed open dense subset in the ambient space. Finally in Section 5 we will give some concluding remarks.

\subsection{Acknowledgements}

I want to thank David Gabai for very useful conversations, and helpful remarks. I want to thank the referee for very valuable remarks.

\section{Preliminaries}

In this section, we will overview the basic results which we use in the following sections.

Definition 2.1 A minimal plane is a plane such that the mean curvature is 0 at every point. A least area plane is a plane such that any disk in the plane has least area among the disks with same boundary. A least area plane is minimal, but the converse is not true in general. 
Definition 2.2 A linear operator between two Banach spaces is called a Fredholm operator if the dimension of the kernel and codimension of the image are finite. The difference between these dimensions is called the Fredholm index of the operator. A map between two Banach manifolds is a Fredholm map if the derivative is a Fredholm operator at every point. A regular value is a point in the image such that for any point in the preimage (may be empty), the derivative is surjective.

The classical theorem of the subject is the Sard-Smale theorem from [17].

Theorem 2.1 (Sard-Smale) Let $f: X \rightarrow Y$ be a Fredholm map. Then the regular values of $f$ are almost all of $Y$, that is, except a set of the first category.

We will use the following spaces in the remaining part of the paper.

$$
\begin{aligned}
A & =\left\{\alpha \in C^{3}\left(S^{1}, S_{\infty}^{2}\right) \mid \alpha \text { embedding }\right\} \\
D & =\left\{u \in C^{3}\left(S^{1}, S^{1}\right) \mid u \text { diffeomorphism and } u\left(e^{2 k \pi i / 3}\right)=e^{2 k \pi i / 3}, k=1,2,3\right\} \\
M & =\left\{f: D^{2} \rightarrow \mathbb{M}^{3} \mid f\left(D^{2}\right) \text { minimal and }\left.f\right|_{\partial D^{2}} \in A\right\}
\end{aligned}
$$

Now, we will summarize the results of [4] which are essential for this paper. In [4], we studied the asymptotic Plateau problem by using global analysis methods. We first looked at the space $M$. We identified the minimal maps in $M$ with the conformal harmonic maps, and considered $M$ as a subspace of the space of harmonic maps. Then by using $\mathrm{Li}$ and Tam's results $[11 ; 12]$, we identified these harmonic maps with their boundary parametrizations, corresponding to the space $A \times D$.

In other words, we realize $M$, the space of minimal planes, as a subspace of the product bundle $A \times D$, by identifying $(\alpha, u)$ in $A$ with its unique harmonic extension $\widetilde{\alpha \circ u}$. Let $\alpha \in A$, and consider the fiber $(\{\alpha\} \times D) \cap M$. This set contains all minimal planes, $\Sigma_{i}={\widetilde{\alpha O u_{i}}}_{i}\left(D^{2}\right)$, with asymptotic boundary $\Gamma=\alpha\left(S^{1}\right)$, that is, $\partial_{\infty} \Sigma_{i}=\Gamma$. Here, the projection map $\pi$ from $A \times D$ into $A$, sends the elements of $M$ to their boundary parametrizations. By using Tomi and Tromba's techniques in [18], we proved that $\left.\pi\right|_{M}$ is Fredholm of index 0 .

Theorem 2.2 $M=\left\{(\alpha, u) \in A \times D \mid \widetilde{\alpha \circ u}\left(D^{2}\right)\right.$ is minimal $\}$ is a submanifold of the product bundle $A \times D$; and the bundle projection map when restricted to $M$, $\left.\pi\right|_{M}: M \rightarrow A$, is Fredholm of index 0 .

Then by using the boundary regularity results of Hardt and Lin [6] to get compactness, we showed that for a generic curve $\Gamma$ in $A$, there are finitely many least area planes in $\pi^{-1}(\Gamma)$. In other words, we proved that for a generic curve, there exist finitely 
many least area planes spanning that curve. Indeed, this is true in more generality. By using the more general regularity results of Tonegawa [19] instead of those of [6], the same technique shows that for a generic curve, there exist finitely many minimal planes spanning that curve.

Theorem 2.3 Let $\Gamma \subset S_{\infty}^{2}\left(\mathbb{M}^{3}\right)$ be a generic curve in $A$. Then there are finitely many minimal planes $\Sigma$ in $\mathbb{H}^{3}$ with $\partial_{\infty} \Sigma=\Gamma$.

Proof In [19, Theorem 1.6], Tonegawa shows that if the asymptotic curve $\Gamma \subset S_{\infty}^{2}\left(\mathbb{M}^{3}\right)$ is $C^{3, \mu}$, and the minimal plane $\Sigma \subset \mathbb{M}^{3}(H=0)$ asymptotic to $\Gamma$, then $\Sigma \cup \Gamma$ is also $C^{3, \mu}$ regular up to the boundary. In [4, Lemma 4.5], we used the same result for least area planes from [14]. If we replace this boundary regularity result for least area planes with the more general result for minimal planes, we see that [4, Lemma 4.5] is true for minimal planes, too. Then this implies by [4, Theorem 4.7], there are finitely many minimal planes for a generic asymptotic curve.

Remark 2.1 With the above notation, this theorem implies that for a generic curve $\alpha \in A, \pi^{-1}(\alpha) \subset M$ is a finite set, that is, generic curves have finitely many preimages in $M$ under the projection map $\pi: M \rightarrow A$.

Remark 2.2 In [4], we took $A$ as the space of $C^{3}$ immersions instead of $C^{3}$ embeddings. In this paper, we are only interested in $C^{3}$ embeddings. Since $A$ is an open submanifold of the space of immersions, we can replace it with the corresponding space in [4], and the same proofs will go through.

The next theorem we use is the classical inverse function theorem for Banach manifolds (see Lang [8]).

Theorem 2.4 (Inverse Function Theorem) Let $M$ and $N$ be Banach manifolds, and let $f: M \rightarrow N$ be a $C^{p}$ map. Let $x_{0} \in M$ and $d f$ be an isomorphism at $x_{0}$. Then $f$ is local $C^{p}$ diffeomorphism, that is, there exists an open neighborhood of $U \subset M$ of $x_{0}$ and an open neighborhood $V \subset N$ of $f\left(x_{0}\right)$ such that $\left.f\right|_{U}: U \rightarrow V$ is $C^{p}$ diffeomorphism.

Now, we can establish the starting point of the paper.

Theorem 2.5 Let $\Gamma \in A$ be a generic curve as above. Then for any $\Sigma \in \pi^{-1}(\Gamma)$, there exist neighborhoods $U_{\Sigma} \subset M$, and $V_{\Gamma} \subset A$ such that $\left.\pi\right|_{U_{\Sigma}}: U_{\Sigma} \rightarrow V_{\Gamma}$ is a homeomorphism. 
Proof By Theorem 2.2, the map $\left.\pi\right|_{M}: M \rightarrow A$ is Fredholm of index 0. By the SardSmale Theorem, the regular values are generic for $\pi$. By the work of Anderson [2], we also know that $\left.\pi\right|_{M}$ is surjective. Let $\Gamma \in A$ be a regular value, and $\Sigma \in \pi^{-1}(\Gamma) \subset M$. Since $\Gamma$ is a regular value, $D \pi(\Sigma): T_{\Sigma} M \rightarrow T_{\Gamma} A$ is surjective. Moreover, we know that $\pi$ is Fredholm of index 0 . This implies $D \pi$ is an isomorphism at the point $\Sigma \in M$. By the Inverse Function Theorem, there exists a neighborhood $U_{\Sigma}$ of $\Sigma$ in $M$, which $\pi$ maps homeomorphically onto a neighborhood $V_{\Gamma}$ of $\Gamma$ in $A$.

\section{Foliated neighborhoods of least area planes}

In this section, our aim is to construct a foliated neighborhood for any least area plane spanning a given generic curve in $A$. Moreover, we will show that the leaves of this foliation are embedded least area planes whose asymptotic boundaries are disjoint from each other. By using this, we will show the uniqueness of the least area plane spanning the generic curve.

We will abuse the notation by using interchangeably the map $\Gamma: S^{1} \rightarrow S_{\infty}^{2}\left(\mathbb{M}^{3}\right)$ with its image $\Gamma\left(S^{1}\right)$. Similarly the same is true for $\Sigma: D^{2} \rightarrow \mathbb{M}^{3}$ and its image $\Sigma\left(D^{2}\right)$.

Let $\Gamma_{0} \in A$ be a generic curve, and let $\Sigma_{0} \in \pi^{-1}\left(\Gamma_{0}\right) \subset M$ be a least area plane whose existence is guaranteed by Anderson's result in [2]. Then by Theorem 2.5, there is a neighborhood of $\Sigma_{0} \in U \subset M$ that is homeomorphic to the neighborhood $\Gamma_{0} \in V \subset A$.

Let $\Gamma:[-\epsilon, \epsilon] \rightarrow V$ be a path such that $\Gamma(0)=\Gamma_{0}$ and for any $t, t^{\prime} \in[-\epsilon, \epsilon], \Gamma_{t} \cap \Gamma_{t^{\prime}}=$ $\varnothing$. In other words, $\left\{\Gamma_{t}\right\}$ foliates a neighborhood of $\Gamma_{0}$ in $S_{\infty}^{2}\left(\mathbb{H}^{3}\right)$. Let $\Sigma_{t} \in U$ be the preimage of $\Gamma_{t}$ under the homeomorphism.

Lemma 3.1 $\left\{\Sigma_{t}\right\}$ is a foliation of a neighborhood of $\Sigma_{0}$ in $\mathbb{U}^{3}$ by embedded least area planes.

Proof We claim that we can assume that $\Sigma_{ \pm \epsilon}$ are least area planes. By Remark 2.1, we know that $\Gamma_{0}$ has finitely many preimages, say $\left\{S_{1}, S_{2}, \ldots, S_{k}\right\} \subset M$. Then by Theorem 2.5, for each $S_{i}$, we have a neighborhood $U_{i} \subset M$ such that $\left.\pi\right|_{U_{i}}: U_{i} \rightarrow V_{i}$ is a homeomorphism where $V_{i} \subset A$ is a neighborhood of $\Gamma_{0}$. Now, by taking the intersection, we can assume $V_{i}=V_{j}$ (say $V$ ) and $\pi^{-1}(V)=\left\{U_{1}, U_{2}, \ldots, U_{k}\right\}$. We also assume that $\left\{\Gamma_{t}\right\} \subset V$. By Anderson's theorem, we know that there exists a least area plane $\Sigma_{t}$ for any $\Gamma_{t}$ with $\partial_{\infty} \Sigma_{t}=\Gamma_{t}$. This means for any $t \in(-\epsilon, \epsilon)$, there exists a least area plane $\Sigma_{t} \in \pi^{-1}\left(\Gamma_{t}\right)$, and it must belong to $U_{i}$ for some $i$. Since there are infinitely many $\Sigma_{t}$, we can find a $U_{i}$ such that there are three least area planes $\Sigma_{t_{0}}, \Sigma_{t_{1}}, \Sigma_{t_{2}}$ in the preimage of $\left\{\Gamma_{t}\right\}$ restricted to $U_{i}$, that is, $\Sigma_{t_{j}} \in U_{i} \cap \pi^{-1}\left(\left\{\Gamma_{t}\right\}\right)$. 
Now let $t_{0}<0 \leq t_{1}<t_{2}$, and we can reparametrize the path $\Gamma$ so that $\Gamma_{-\epsilon}=\Gamma_{t_{0}}$, $\Gamma_{0}=\Gamma_{t_{1}}, \Gamma_{+\epsilon}=\Gamma_{t_{2}}$. From now on, we will assume that $\Sigma_{ \pm \epsilon}$ are also least area planes.

We will prove the lemma in three steps.

Step 1 For any $s \in[-\epsilon, \epsilon], \Sigma_{s}$ is an embedded plane.

Since $\Sigma_{0}$ is a least area plane, by [4, Theorem 4.5], $\Sigma_{0}$ is an embedded plane. Now, $\left\{\Sigma_{t}\right\}$ is a continuous family of minimal planes. We cannot apply the same theorem to these planes, since the theorem is true for least area planes, while our planes are only minimal.

Let $s_{0}=\inf \left\{s \in(0, \epsilon] \mid \Sigma_{s}\right.$ is not embedded $\}$. However, since $\left\{\Sigma_{t}\right\}$ is continuous family of planes, and this can only happen when $\Sigma_{s_{0}}$ has tangential self intersection (locally lying on one side), this contradicts with the maximum principle for minimal surfaces. So for all $s \in[0, \epsilon], \Sigma_{s}$ is embedded. Similarly, this is true for $s \in[-\epsilon, 0]$, and the result follows.

Step $2\left\{\Sigma_{t}\right\}$ is a foliation, that is, for any $t, t^{\prime} \in[\epsilon, \epsilon], \Sigma_{t} \cap \Sigma_{t^{\prime}}=\varnothing$.

Assume on the contrary that there exist $t_{1}<t_{2}$ such that $\Sigma_{t_{1}} \cap \Sigma_{t_{2}} \neq \varnothing$. First, since the asymptotic boundaries $\Gamma_{t_{1}}$ and $\Gamma_{t_{2}}$ are disjoint, the intersection cannot contain an infinite line. So the intersection must be a collection of closed curves. We will show that in this situation, there must be a tangential intersection between two planes, and this will contradict to the maximum principle for minimal surfaces.

If $\Sigma_{t_{2}}$ does not intersect all the minimal planes $\Sigma_{s}$ for $s \in\left[-\epsilon, t_{2}\right]$, let

$$
s_{0}=\sup \left\{s \in\left[-\epsilon, t_{2}\right] \mid \Sigma_{t_{2}} \cap \Sigma_{s}=\varnothing\right\} .
$$

Then, since $\left\{\Sigma_{t}\right\}$ is a continuous family of minimal planes, it is clear that $\Sigma_{t_{2}}$ must intersect $\Sigma_{s_{0}}$ tangentially, and lie in one side of $\Sigma_{s_{0}}$. However, this contradicts with the maximum principle for minimal surfaces.

So, let's assume $\Sigma_{t_{2}}$ intersects all minimal planes $\Sigma_{s}$ for $s \in\left[-\epsilon, t_{2}\right]$. Let

$$
s_{0}=\sup \left\{s \in\left(-\epsilon, t_{2}\right] \mid \Sigma_{-\epsilon} \cap \Sigma_{s}=\varnothing\right\} .
$$

If there exists an $s_{0} \in\left(-\epsilon, t_{2}\right]$, then again since $\left\{\Sigma_{t}\right\}$ is a continuous family of minimal planes, $\Sigma_{s_{0}}$ must intersect $\Sigma_{-\epsilon}$ tangentially, and lie in one side of $\Sigma_{s_{0}}$. However, this contradicts with the maximum principle for minimal surfaces.

If there is no such $s_{0} \in\left(-\epsilon, t_{2}\right]$, then this means $\Sigma_{0} \cap \Sigma_{-\epsilon} \neq \varnothing$. Since $\partial_{\infty} \Sigma_{0}=\Gamma_{0}$ is disjoint from $\partial_{\infty} \Sigma_{-\epsilon}=\Gamma_{-\epsilon}$ in $S_{\infty}^{2}\left(\mathbb{H}^{3}\right)$, the intersection cannot contain an infinite line. So, we can find a simple closed loop $\gamma$ in the intersection $\Sigma_{0} \cap \Sigma_{-\epsilon}$. But, since 
$\Sigma_{0}$ and $\Sigma_{-\epsilon}$ are both least area planes, this is a contradiction by the Meeks-Yau exchange roundoff trick.

Step 3 For any $s \in[-\epsilon, \epsilon], \Sigma_{s}$ is a least area plane.

Fix $\Sigma_{s}$ for $s \in(-\epsilon, \epsilon)$. We will show that any subdisk of $\Sigma_{s}$ is a least area disk. Let $\left[\Sigma_{-\epsilon}, \Sigma_{\epsilon}\right]$ be the region bounded by embedded planes $\Sigma_{-\epsilon}$ and $\Sigma_{\epsilon}$ in $\mathbb{T}^{3}$. By above results, $\Sigma_{s} \subset\left[\Sigma_{-\epsilon}, \Sigma_{\epsilon}\right]$. Let $\gamma \subset \Sigma_{s}$ be an extreme (in the boundary of its convex hull) simple closed curve. By Morrey's theorem [16], there exists a least area disk $D$ with $\partial D=\gamma$. By the work of Anderson [1], the least area disks in hyperbolic space have convex hull property, so $D$ is in its convex hull. Since we chose $\gamma$ to be extreme, by Meeks and Yau's theorem [15], $D$ is a least area embedded disk in $\mathbb{H}^{3}$ spanning $\gamma$.

We claim that $D \subset\left[\Sigma_{-\epsilon}, \Sigma_{\epsilon}\right]$. Otherwise, $D$ must intersect one or both of the planes. Since the boundary of $D$, is disjoint from $\Sigma_{ \pm \epsilon}$, then there must be a simple closed curve in the intersection. But all of them are least area, and this is a contradiction by exchange roundoff trick of Meeks and Yau.

Now, we want to show that $D \subset \Sigma_{s}$. If $D$ is not contained in $\Sigma_{s}$, it must intersect other leaves nontrivially. Since $D$ is completely in the domain $\left[\Sigma_{-\epsilon}, \Sigma_{\epsilon}\right]$, then $\left\{\Sigma_{t}\right\} \cap D$ induce a singular 1-dimensional foliation $F$ on $D$. The singularities of the foliation are isolated, as $\left\{\Sigma_{t}\right\}$ are minimal planes. Since Euler characteristic of the disk is 1, by the Poincaré-Hopf index formula there must be a positive index singularity implying tangential (lying on one side) intersection of $D$ with some leave $\Sigma_{s}$. However, this contradicts with the maximum principle for minimal surfaces. So, we show that the subdisks of $\Sigma_{s}$ with extreme boundary are least area disks. But since for any subdisk of $\Sigma_{s}$, we can find a bigger disk containing it with an extreme boundary, it is true for any subdisk of $\Sigma_{s}$. This shows that for any $s \in(-\epsilon,+\epsilon), \Sigma_{s}$ is a least area plane.

Lemma 3.2 Let $\Gamma$ be a generic curve as described above. Then, there exists a unique least area plane $\Sigma$ with $\partial_{\infty} \Sigma=\Gamma$.

Proof We will use the notation of the proof of the previous lemma. In the proof of the previous lemma, we constructed a foliation by least area planes $\left\{\Sigma_{t}\right\}$ with asymptotic boundaries $\left\{\Gamma_{t}\right\}$. After the reparametrization in the beginning of the proof of the previous lemma, the generic curve $\Gamma$ becomes an interior leaf of the foliation $\left\{\Gamma_{t}\right\}$. In other words, there is a $t^{\prime} \in(-\epsilon, \epsilon)$ such that $\Gamma=\Gamma_{t^{\prime}}$. Since $t^{\prime}$ is an interior point, $\left\{\Sigma_{t}\right\}$ is also foliation for a neighborhood of $\Sigma_{t^{\prime}}$.

Let $\widehat{\Sigma}$ be another least area plane with asymptotic boundary $\Gamma_{t^{\prime}}$. If $\widehat{\Sigma} \neq \Sigma_{t^{\prime}}$ then $\widehat{\Sigma}$ must intersect a leave in the foliated neighborhood of $\Sigma_{t^{\prime}}$, say $\Sigma_{s}$. But, since $\partial_{\infty} \Sigma_{s}=\Gamma_{s}$ is disjoint from $\partial_{\infty} \Sigma_{t^{\prime}}=\Gamma_{t^{\prime}}$, this implies the intersection cannot have 
infinite lines, but closed loops. However, these are least area planes, and by the MeeksYau exchange roundoff trick, two least area planes cannot intersect in a closed loop. This is a contradiction.

Remark 3.1 This lemma does not say that there exists a unique minimal plane spanning a given generic curve. In the proof of the lemma, we essentially use the plane being least area.

Hence, we have proved the following theorem:

Theorem 3.3 Let $\Gamma$ be a generic curve in $A$. Then there exists a unique least area plane $\Sigma$ in $\mathbb{H}^{3}$ with $\partial_{\infty} \Sigma=\Gamma$.

So far we have proved the uniqueness of least area planes for a subset $\widehat{A} \subset A$ which is except a first category set. In the next section, we will show that this is true for a more general class of curves, that is, an open dense subset of $A$.

\section{Open dense set of curves}

In this section, we will show that any generic curve, which is a regular value of the Fredholm map $\pi$, has an open neighborhood such that the uniqueness result holds for any curve in this neighborhood.

Let $\Gamma_{0} \in A$ be a generic curve, and let $\Sigma_{0} \in \pi^{-1}\left(\Gamma_{0}\right) \subset M$ be the unique least area plane spanning $\Gamma_{0}$. Let $U \subset M$ be the neighborhood of $\Sigma_{0}$ homeomorphic to the neighborhood $V \subset A$ of $\Gamma_{0}$ as in the previous section. We will show that $\Gamma_{0}$ has a smaller open neighborhood $V^{\prime} \subset V$ such that for any $\Gamma \in V^{\prime}$, there exists a unique least area plane in $\mathbb{H}^{3}$ with $\partial_{\infty} \Sigma=\Gamma$.

First we will show that the curves disjoint from $\Gamma_{0}$ in the open neighborhood also bounds a unique least area plane in $\mathbb{H}^{3}$.

Lemma 4.1 Let $\beta \in V$ with $\beta \cap \Gamma_{0}=\varnothing$. Then there exists a unique least area plane $\Sigma$ in $\mathbb{M}^{3}$ with $\partial_{\infty} \Sigma=\beta$.

Proof Since $\beta \in V$ is disjoint from $\Gamma_{0}$, we can find a path $\Gamma:(-\epsilon, \epsilon) \rightarrow V$, such that $\left\{\Gamma_{t}\right\}$ foliates a neighborhood of $\Gamma_{0}$ in $S_{\infty}^{2}\left(\mathbb{H}^{3}\right)$, and $\beta$ is one of the leaves, that is, $\beta=\Gamma_{s}$ for some $s \in(-\epsilon, \epsilon)$. Then the proofs of the previous section implies that $\Sigma_{\beta}=\Sigma_{s}$ and $\left\{\Sigma_{t}\right\}$ also gives a foliation of a neighborhood of $\Sigma_{\beta}$ by least area planes. Then proof of Lemma 3.2 implies that $\Sigma_{\beta}$ is the unique least area plane spanning $\beta$. 
Now, if we can show the same result for the curves in $V$ intersecting $\Gamma_{0}$, then we are done. Unfortunately, we cannot do that, but we will bypass this by going to a smaller neighborhood.

Lemma 4.2 There exists a neighborhood $V^{\prime} \subset V$ of $\Gamma_{0}$ such that for any $\Gamma_{0}^{\prime} \in V^{\prime}$, there exists a unique least area plane with asymptotic boundary $\Gamma_{0}^{\prime}$.

Proof Let $\beta_{1}, \beta_{2} \in V$ be two Jordan curves in $S_{\infty}^{2}\left(\mathbb{M}^{3}\right)$ disjoint from $\Gamma_{0}$ and lying in opposite side of $\Gamma_{0}$ in $S_{\infty}^{2}\left(\mathbb{H}^{3}\right)$. Then, $\beta_{1}$ and $\beta_{2}$ bounds an open annulus $B$ in $S_{\infty}^{2}\left(\mathbb{M}^{3}\right)$, where $\Gamma_{0}$ is in the interior of $B$. Then, let $V^{\prime}=\{\Gamma \in V \mid \Gamma \subset B\}$ be an open neighborhood of $\Gamma_{0}$.

Now, fix $\Gamma_{0}^{\prime} \in V^{\prime}$. By the assumption on $V^{\prime}, \Gamma_{0}, \Gamma_{0}^{\prime}$ both belong to the annulus $B \subset S_{\infty}^{2}\left(\mathbb{H}^{3}\right)$. Then, we can find two paths $\Gamma, \Gamma^{\prime}:[-\epsilon, \epsilon] \rightarrow V$ where $\left\{\Gamma_{t}\right\},\left\{\Gamma_{t}^{\prime}\right\}$ foliate $B$ such that $\Gamma(\epsilon)=\Gamma^{\prime}(\epsilon)=\beta_{1}, \Gamma(-\epsilon)=\Gamma^{\prime}(-\epsilon)=\beta_{2}$, and $\Gamma(0)=\Gamma_{0}$, $\Gamma^{\prime}(0)=\Gamma_{0}^{\prime}$.

By Lemma 3.1, we know that $\left\{\Gamma_{t}\right\}$ induces $\left\{\Sigma_{t}\right\}$ family of embedded least area planes with asymptotic boundary $\left\{\Gamma_{t}\right\}$. Moreover, these least area planes are unique with the given asymptotic boundary, and the leaves of the foliation in the neighborhood of $\Sigma_{0}$.

Now, consider the preimage of the path $\Gamma^{\prime}$ under the homeomorphism $\left.\pi\right|_{U}: U \rightarrow V$. This will give us a path $\Sigma^{\prime} \subset U \subset M$, which is a continuous family of minimal planes, say $\left\{\Sigma_{t}^{\prime}\right\}$. We claim that this is also a family of embedded least area planes inducing a foliated neighborhood of $\Sigma_{0}^{\prime}$. By previous section, we can also assume that $\beta_{1}$ and $\beta_{2}$ bound a unique least area plane, say $\partial_{\infty} \Sigma_{\epsilon}=\beta_{1}$ and $\partial_{\infty} \Sigma_{-\epsilon}=\beta_{2}$. This means $\Sigma_{ \pm \epsilon}^{\prime}=\Sigma_{ \pm \epsilon}$. So, the family $\left\{\Sigma_{t}^{\prime}\right\}$ has embedded least area planes $\Sigma_{ \pm \epsilon}^{\prime}$. Then a slight modification of the proof of Lemma 3.1 shows that $\left\{\Sigma_{t}^{\prime}\right\}$ is a family of embedded least area planes inducing a foliation of a neighborhood of $\Sigma_{0}^{\prime}$. By Lemma 3.2, $\Sigma_{0}^{\prime}$ is the unique least area plane spanning $\Gamma_{0}^{\prime}$.

Thus, we get the following theorem.

Theorem 4.3 There exists an open dense subset $A^{\prime}$ in $A$, where

$$
A=\left\{\alpha \in C^{3}\left(S^{1}, S_{\infty}^{2}\right) \mid \alpha \text { embedding }\right\}
$$

with $C^{3}$ topology, such that for any $\Gamma \in A^{\prime}$, there exists a unique least area plane with asymptotic boundary $\Gamma$.

Proof The set of regular values of Fredholm map, say $\hat{A}$, is the whole set except a set of the first category by the Sard-Smale theorem. So, the regular curves are dense in 
$A$. By the above lemmas, for any regular curve $\Gamma_{0}$, there exists an open neighborhood $V_{\Gamma_{0}}^{\prime} \subset A$ which the uniqueness result holds. So, $A^{\prime}=\bigcup_{\Gamma \in \widehat{A}} V_{\Gamma}^{\prime}$ is an open dense subset with the desired properties.

\section{Final remarks}

\subsection{The technique}

The technique we used in this paper to show uniqueness of the least area planes is similar to the techniques of Anderson and Lin for their proofs for convex domains [1] and star shaped domains [14] in $S_{\infty}^{2}\left(\mathbb{H}^{3}\right)$. The idea is simple. Fix the least area plane. Construct a neighborhood of the least area plane foliated by least area planes with disjoint asymptotic boundaries. Then any other least area plane with same asymptotic boundary with the original one must intersect the other least area planes. But this intersection cannot contain an infinite line as the asymptotic boundaries are disjoint. So the intersection must be a collection of closed curves. But the Meeks-Yau exchange roundoff trick says that two least area planes cannot intersect in a closed loop, and gives the desired contradiction.

On the other hand, hyperbolic space and its asymptotic boundary are not very essential for this technique. One can employ the same method for any convex domain, and least area disks whose boundaries are in the boundary of this convex domain, once one has a continuous family of minimal disks.

\subsection{Extremal curves in $\mathbb{R}^{3}$}

As we mentioned above, we can use this technique for extremal curves in convex domains in $\mathbb{R}^{3}$. Let $N \subset \mathbb{R}^{3}$ be a smooth convex domain. Then by changing the space of curves from embeddings of $S^{1}$ into $\mathbb{R}^{3}$ to the embeddings of $S^{1}$ into $\partial N$ in the paper by Tomi and Tromba [18], the whole proof would go through. So this will establish the necessary analytical background to employ the techniques in this paper. Then one can fix a regular extremal curve in $\partial N$, and get a foliated neighborhood of the embedded least area disk spanning the fixed curve in $N$. This proves again the uniqueness of the least area disk. So, one can show that for a given smooth convex domain $N$ in $\mathbb{R}^{3}$, there exists an open dense subset of the Jordan curves in $\partial N$ which bound a unique least area disk in $\mathbb{R}^{3}$. In some sense, this is generic uniqueness for extremal curves in $\mathbb{R}^{3}$.

By using the same setting and similar techniques, Fang-Hua Lin showed [13] that an extremal curve either bounds a unique least area disk, or two "extremal" stable minimal 
disks. One can get the same result by using the methods of this paper. Also, by using similar techniques in an analytical way, Li-Jost proved [10] that a $C^{3, \mu}$ Jordan curve in $\mathbb{H}^{3}$ with total curvature less than $4 \pi$ bounds a unique least area disk.

\subsection{Questions}

As we mentioned in the introduction, there are not many results on the number of solutions of asymptotic Plateau problem in $\mathbb{H}^{3}$. In this paper, we showed a generic uniqueness result for some smooth class of curves. One suspects whether there is any smooth class of Jordan curves in $S_{\infty}^{2}\left(\mathbb{M}^{3}\right)$ such that any curve in this class bounds only finitely many least area planes. Another question in the opposite direction is whether there is any smooth Jordan curve in $S_{\infty}^{2}\left(\mathbb{M}^{3}\right)$ bounding infinitely many least area planes.

If you remove the condition being plane (the topological type of the surface is disk), Anderson gave examples of Jordan curves bounding infinitely many area minimizing surfaces in [2]. If one remove the embeddedness condition for the curve, the universal cover of a hyperbolic manifold fibering over a circle, induces a Peano curve in $S_{\infty}^{2}\left(\mathbb{M}^{3}\right)$ bounding infinitely many minimal planes, corresponding to the universal cover of the least area representative of a fiber.

It is still an open question to find a Jordan curve bounding infinitely many least area planes. But it is reasonable to hope that the bridge principle holds in this case too, and one constructs a rectifiable embedded curve in $S_{\infty}^{2}\left(\mathbb{M}^{3}\right)$ similar to the Euclidean case.

\section{References}

[1] M T Anderson, Complete minimal varieties in hyperbolic space, Invent. Math. 69 (1982) 477-494 MR679768

[2] M T Anderson, Complete minimal hypersurfaces in hyperbolic n-manifolds, Comment. Math. Helv. 58 (1983) 264-290 MR705537

[3] B Coskunuzer, Uniform 1-cochains and genuine laminations arXiv: math.GT/0304094

[4] B Coskunuzer, Minimal planes in hyperbolic space, Comm. Anal. Geom. 12 (2004) 821-836 MR2104077

[5] D Gabai, On the geometric and topological rigidity of hyperbolic 3-manifolds, J. Amer. Math. Soc. 10 (1997) 37-74 MR1354958

[6] R Hardt, F-H Lin, Regularity at infinity for area-minimizing hypersurfaces in hyperbolic space, Invent. Math. 88 (1987) 217-224 MR877013 
[7] J Hass, P Scott, The existence of least area surfaces in 3-manifolds, Trans. Amer. Math. Soc. 310 (1988) 87-114 MR965747

[8] S Lang, Real analysis, Addison-Wesley, Reading, MA (1983) MR783635

[9] U Lang, The asymptotic Plateau problem in Gromov hyperbolic manifolds, Calc. Var. Partial Differential Equations 16 (2003) 31-46 MR1951491

[10] X Li-Jost, Uniqueness of minimal surfaces in Euclidean and hyperbolic 3-space, Math. Z. 217 (1994) 275-285 MR1296397

[11] P Li, L-F Tam, Uniqueness and regularity of proper harmonic maps, Ann. of Math. (2) 137 (1993) 167-201 MR1200080

[12] P Li, L-F Tam, Uniqueness and regularity of proper harmonic maps II, Indiana Univ. Math. J. 42 (1993) 591-635 MR1237061

[13] F-H Lin, Plateau's problem for H-convex curves, Manuscripta Math. 58 (1987) 497 511 MR894867

[14] F-H Lin, On the Dirichlet problem for minimal graphs in hyperbolic space, Invent. Math. 96 (1989) 593-612 MR996556

[15] W H Meeks III, S T Yau, The classical Plateau problem and the topology of threedimensional manifolds., Topology 21 (1982) 409-442 MR670745

[16] C B Morrey Jr, The problem of Plateau on a Riemannian manifold, Ann. of Math. (2) 49 (1948) 807-851 MR0027137

[17] S Smale, An infinite dimensional version of Sard's theorem, Amer. J. Math. 87 (1965) 861-866 MR0185604

[18] F Tomi, A J Tromba, Extreme curves bound embedded minimal surfaces of the type of the disc, Math. Z. 158 (1978) 137-145 MR486522

[19] Y Tonegawa, Existence and regularity of constant mean curvature hypersurfaces in hyperbolic space, Math. Z. 221 (1996) 591-615 MR1385170

Department of Mathematics, Yale University

New Haven CT 06520, USA

baris.coskunuzer@yale.edu

Proposed: Gang Tian

Received: 5 August 2004

Seconded: Tobias Colding, David Gabai

Revised: 23 February 2006 